\title{
Sudden unexpected death in epilepsy: mechanisms, risk factors and the role of the nurse
}

\author{
Deborah L Brennan and Andrew D Powell \{AQ1: Is author order correct?\}
}

\begin{abstract}
Epilepsy is a disease which can have significant effects upon a person's life. A subject discussed less frequently is sudden unexpected death in epilepsy (SUDEP) \{AQ2: Edits to sentence OK? D s article summarises the main risk factors for its occurlence; notably, seizure types, medications management and sleeping position. It discusses potential pathophysiological mechanisms, including the emerging research on laryngospasm and the role that genetics may play in (7) easing risk. Finally, it identifies several nursing responsibilities 2.r. in the structure of a SUDEP checklist that has been shown to improve patient outcomes. It discusses how these responsibilities are underpinned by patient education and the need for all nurses to develop their own knowledge of epilepsy and SUDEP to facilitate this.
\end{abstract}

Key Words epilepsy; SUDEP; pathophysiology; nursing responsibilities; risk factors; education

Authors Deborah L Brennan, School of Nursing, Birmingham City University; Andrew D Powell, School of Health Sciences, Birmingham Citv University \{AQ3: Are affiliations correct, and do you wish job titles to appear?

Correspondence andy.powell@bcu.ac.uk

Accepted February 2019

This article has been subject to double-blind peer review.

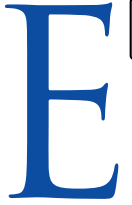
(11) psy is a disease of the brain and "exists in a patient who has had a seizure and whose brain, for whatever reason, demonstrates a pathologic and enduring tendency to have recurrent seizures (Fisher et al, 2014 \{AQ4: Referencing style has been changed throughout. Please check that this has been done correctly\}). In the UK, epilepsy is estimated to affect approximately 400000 people, with most cases well controlled with antiepileptic drugs (AEDs) (National Institute for Health and Care Excellence (NICE), 2018).

Epilepsy can have a profound impact on a person's quality of life (QoL) and continues to be stigmatised in society, often because of poor understanding by the general population (Mula and Sander, 2016). Levels of depression and anxiety are high in those living with epilepsy, with the psychosocial difficulties that result from living with epilepsy potentially having a greater impact than the disease itself (Mula and Sander, 2016). Suicide rates are twice as high in those living with epilepsy as in the general population, and three times as high $\{$ AQ5: Edit OK? Wen the disease is accompanied by psychiatric co-mororaity (Mula and Sander, 2016). The side effects of medications used to manage seizures also (mact QoL negatively (Fisher et al, 2015).

The negative impact that epilepsy exerts on an individual's QoL is linked with poor mental health, poor outcomes and an increased risk of sudden unexpected death in epilepsy (SUDEP) (NICE, 2015). Although uncommon, SUDEP is a leading cause of premature mortality in epilepsy (Ellis and Szabo, 2018). This article examines SUDEP, including risk factors, pathophysiology and the role of the nurse in reducing risk through improving how people live with epilepsy. It aims to present nurses with $\&$ wledge that develops and clarifies understanding cru develops \{AQ6: Can we replace this second 'develops' with 'improves' $D$ eir practise.

\section{What is SUDEP?}

SUDEP is defined as 'sudden, unexpected, witnessed or unwitnessed, non-traumatic and non-drowning death, in an individual with epilepsy, with or without evidence for a seizure and excluding documented status epilepticus, in which post mortem does not reveal a cause of death' (Nashef et al, 2012). In addition, a nearSUDEP event is one in which 'cardiorespiratory arrest was reversed by resuscitation efforts with subsequent survival for more than 1 hour that has no structural cause' (Nashef et al, 2012).

\section{Incidence rates and prevalence}

Neurological-related deaths have increased by $39 \%$ since 2001, with epilepsy the third-most common cause. Since 2014 , there has been a $9 \%$ increase in epilepsy mortality, with a mean age of death of 70 years; epilepsy-related deaths are three times more likely to occur in deprived areas, with an occurrence rate of 13 per 100000 population (Public Health England, 2018).

Every person with epilepsy is at risk of SUDEP, 
h has an estimated incidence rate of 1.16 cases per I00 population or 0.81 per 100000 population \{AQ7: Please review. The arithmetic does not make sense to me?\} (Thurman et al, 2014). However, the number of deaths is likely to be an underestimate, as many \{AQ8: Do you mean that in many cases SUDEP is not recorded as the cause of death? 2ate not recorded as the cause of death (Middleton et al, 2018). This underreporting may result from the unwitnessed nature of many cases, with a lack of awareness and knowledge by medics and coroners to certify deaths in this way (Flannery and Lomke, 2018).

\section{Risk factors}

As all individuals with epilepsy are at risk of, but may not die from, SUDEP, a greater understanding of the associated risk factors is important. This applies to the individual and their care networks, but also the professionals responsible for the management of their epilepsy and overall health. A large number of SUDEP risk factors have been proposed, with the most common causes being:

- Generalised tonic-clonic seizures (Shankar et al, 2018)

- Poor seizure management and subsequent high seizure frequency (Shankar et al, 2018)

- Poor AED compliance (NICE, 2018; Shankar et al, 2018)

- AED polypharmacy (Shankar et al, 2018)

- Nocturnal seizures (Shankar et al, 2018; Watkins et al, 2018)

(19) $\mathrm{hg}$ in bed during a seizure, particularly if sleeping III the prone position (Shankar et al, 2018; Watkins et al, 2018@

Pther potential risk factors include: time since uragnosis (especially when diagnosed in childhood) (Shankar et al, 2018; Watkins et al, 2018) and intellectual disability (Watkins et al, 2018).

Key to management of these risks factors is an understanding that the risk profile evolves over time, requiring continual dialogue between patient and healthcare professionals, using a patient-centred approach to identify and mitigate (where possible) individual risk (Shankar et al, 2018).

\section{Genetics and SUDEP}

Why certain people are at increased risk, but may not (11) mately die from SUDEP, is unclear $\{A Q 9$ : Why is it und that a person is at increased risk of something, but succumbs to something else?\}. Certain gene mutations increase the risk (B) gnall, 2017), but similar to other aspects of SUD the lack of clear confirmation \{AQ10: Edit OK $\{$ cause of death has hampered the identification of possible genetic factors that contribute to an individual's risk of SUDEP (B) gnall, 2017).

SUDEP does not show familial mmrmeritance; however, several candidate genes have been identified that increase SUDEP risk. Genome analysis of brain tissue from confirmed SUDEP cases has identified genetic variants, including genes responsible for regulating synaptic signalling and ion channels responsible $\{A Q 11$ : do you mean genes responsible for io pannels which affect the cardiac action potential? 2 r the cardiac action potential (Friedman et al, 2018). This provides a plausible link between cardiovascular dysfunction and (1) DEP (Tiron et al, 2015). Of particular importance are genes that can lead to long QT syndrome, which can result in cardiac arrhythmias and potentially sudden death (Tester and Ackerman, 2014. Detailed analysis of the genetic factors that impact an individual's overall SUDEP risk is beyond the scope of this article, but these have been reviewed elsewhere (Bagnall et al, 2017).

Identification of genetic risk factors may not permit treatment of the causes of SUDEP, but awareness should permit more effective management of individual risk. For example, medications, such as antidepressants, antibiotics and anti-psychotics increase the risk of sudden death in individuals with long QT syndrome (Cubeddu, 2016). Due to the relative rarity of $S \cap E P$ events, combined with potential under-rep 2 ng (Middleton et al, 2018), it remains unclear whether certain classes of medication alter an individual's overall risk.

To ensure effective management of the SUDEP risk, further research into potential genetic mutations linked to SUDEP is necessary. This requires rapid identification of SUDEP so that post mortem blood samples are available for all cases, to allow a full genetic screen (Bagnall et al, 2017).

\section{Pathophysiology of SUDEP}

The precise mechanisms that underlie SUDEP remain elusive, largely due to the highly unpredictable and unwitnessed nature of the event. Despite this, a small number of witnessed SUDEP and near-SUDEP events, combined with a greater understanding from animal studies, suggest that a SUDEP event activates an autonomic response that ultimately results in death.

The possible mechanisms leading to a SUDEP event have been broadly categorised into cardiovascular causes and respiratory causes, although it is likely that any death is multifactorial.

\section{Cardiovascular system}

Cardiovascular dysfunction, particularly cardiac arrhythmias, occurs during/after seizures. Sinus tachycardia is common, but other arrhythmias also occur Dlagapudi, 2012). The MORTality in Epilepsy Monturng Unit Study (MORTEMUS), which included 11 definite SUDEP cases, showed sudden death resulted from respiratory and arousal system dysfunction, and bradycardia in postictal periods. Some patients with SUDEP had transient tachyarrhythmias, but these episodes were always followed by bradycardia and asystole (Ryvlin et al, 2013). The link between 
susceptibility to SUDEP and cardiovascular function has been examined and individuals who died from SUDEP had a greater degree of autonomic dysfunction (in) a lower heart rate variability while awake Myers et a1, 2018).

\section{Respiratory system: can seizure-driven laryngospasm explain SUDEP?}

A plausible mechanism for a SUDEP event is a seizure-driven occlusion of the airways, possibly due to laryngospasm. Although a defining characteristic of SUDEP is a lack of definite cause (Nashef et al, 2012), circumstantial evidence for laryngospasm as a precipitating factor is provided by post mortem examinations. Pulmonary oedema is a common feature, occurring in more than half of cases (Kennedy et al, 2015). Respiratory effort against a closed airway, as would occur during laryngospasm, can increase pulmonary capillary pressures resulting in oedema (Ead, 2003).

Support for laryngospasm as a precipitating factor for SUDEP is provided by animal models (Nakase et al, 2016; Stewart et al, 2017; Budde et al, 2018) and clinical case reports (Tavee and Morris, 2008; Lacuey et al, 2018). Animal studies show that seizure activity causing full airway obstruction results in a sudden death, but airway protection prevents death (Nakase et al, 2016). Associated with the laryngospasm was a severe bradycardia and $\subseteq$ changes. In contrast, central apnoea (no airflow, no respiratory effort) did not result in death (Nakase et al, 2016; Stewart et al, 2017).

Two main theories have been suggested for how seizure activity can lead to laryngospasm. Firstly, seizure activity activates recurrent laryngeal nerve tracts, which leads to laryngospasm and closure of the airway (Stewart et al, 2017). An alternative theory proposes that the laryngospasm results from inappropriate activation of a protective reflex (Budde et al, 2018). A common trigger for non-seizure-related laryngospasm is gastro-oesophageal reflux disease (GORD), which occurs due to oesophageal sphincter weakening and acid leaking into the oesophagus (Kavitt and Vaezi, 2013). A reflex results in laryngospasm to prevent the acid from entering the respiratory system (Loughlin et al, 1996). Decreased oesophageal $\mathrm{pH}$ occurs prior to seizure-induced terminal apnoea, while stopping acid from $\{A Q 12$ : Edit to prevent repeated w OK? entering the oesophagus prevented the terminalapnoea, despite similar seizure activity (Budde et al, 2018).

SUDEP and GORD have increased incidence at night; nocturnal seizures are a major risk factor for SUDEP (Shankar et al, 2018; Watkins et al, 2018), while GORD patients report an increase in symptomology \{AQ13: Can symptomology in ro se? 'symptoms'?\}, presumably due to the ease with which acid can enter the oesophagus while recumbent (Kavitt and Vaezi, 2013). In nocturnal GORD, the laryngospasm may be resolved by the individual sitting up, allowing acid to drain from the oesophagus; in contrast, during an unwitnessed seizure-driven laryngospasm, the individual remains unconscious, resulting in terminal apnoea (Budde et al, 2018).

Clinical cases supporting the implication of severe laryngospasm are now emerging. Tavee and co-workers (2008) reported a case of near SUDEP that was preceded by a laryngospasm; importantly, no alterations in the patient's cardiac function were reported. Lacuey et al (2018), reported a video-EEG captured seizure that resulted in increased inspiratory effort, visible tracheal movements and audible inspiratory stridor. This was associated with a rapid reduction in oxygen saturations and persistent severe hypoxemia.

\section{Potential treatment strategies for individuals with SUDEP}

Given the unpredictable nature of SUDEP, reducing the risk for susceptible individuals is of paramount importance. A recent Cochrane review (Maguire et al, 2016) identified various strategies that could be used to minimise the risk of a SUDEP event, but concluded that more research was required to evaluate the effectiveness of any intervention. Strategies included:

- Reduction of the overall risk to patients with epilepsy through surgery, effective seizure management and education

- Detection of the clinical manifestations of seizure (cardiorespiratory arrest etc.) through either observations or via monitoring devices, which might ensure the individual receives supportive interventions to prevent death

- Reduction of the risk through safety devices such as safety pillows which permit airflow

- Pharmacological interventions targeting serotonergic (5-HT), adenosine and opioid signalling pathways.

Due to the key role that the neurotransmitters and neuromodulators serotonin, adenosine and opioids play in the control of respiratory rate (Ackland et al, 2007; Pattinson, 2008), the potential impact of modulation of endogenous levels of these substances on SUDEP risk has been explored. Serotoninergic \{AQ14: serotor Cry neurotransmission underpins respiratory contro, with serotonergic neurons being postulated as the central chemoreceptors that stimulate increases in breathing rate (Teran et al, 2014). It is therefore plausible to speculate that reductions in serotonergic signalling could underpin the changes in respiratory effort that are a hallmark of SUDEP. Animal studies that modulate the extent of serotonergic transmission demonstrate severe apnoea, decreased resnonses to hypercapnia and issues arousing $\{\mathrm{AQ} 15$ : 'is 2 with arousal', or 'arising from'?\} from sleep (Hodges et al, 2009). Additionally, these animals also demonstrate a reduced seizure threshold (Buchanan et al, 2014). Consequently, it was postulated that selective serotonin reuptake inhibitors 
(SSRIs) may prove beneficial to epileptic individuals \{AQ16: I think 'people with (Plepsy', or similar, is now preferred?\}. SSRIs have shbwr clinical effectiveness in the treatment of a variety of diseases where altered serotonergic signalling has been implicated, such as depression (Cipriani et al, 2018). In seizure-susceptible mice, the overall level of respiratory arrest and death was reduced by treatment with the SSRI, fluoxetine (Tupal and Faingold, 2006). Similar results were found in SSRI-treated epileptic patients $\{A Q 1 \bigcirc$ patients with epilepsy'\} who demonstrated reduced oxygen desaturation, although these benefits were limited to patients with partial seizures and did not benefit patients with generalised seizures (Bateman et al, 2010). This observation questions the applicability of SSRIs to reduce SUDEP risk where poorly controlled generalised tonic-clonic seizures are a major risk factor (Shankar et al, 2018).

Other proposed pharmacotherapies include modulation of adenosine and opioids levels. It has been established that seizures can induce release of endogenous opioid from brainstem nuclei (Hammers et al, 2007). Given the sensitivity of the respiratory system to opioids, as highlighted by the major life-threatening side effect of respiratory depression (Pattinson, 2008), it has been proposed that administration of the opioid antagonist naloxone may reverse postictal hypoxemia (Rheims et al, 2016).

\section{Reduction of the likelihood of SUDEP occurring: nursing responsibilities $\{A Q \&$ Edit to heading OK?}

While the risk of SUDEP can never fully be eliminated, steps can be taken to reduce it, and this process should begin when a person is first diagnosed with epilepsy. Best practice guidance by NICE in the UK, states that information should \{AQ19: EdiST? be provided to all individuals with epilepsy, Including their care network, regarding SUDEP. General information about epilepsy, medications and risk management should be provided not only at the time of their diagnosis, but as ongoing provision. Best practise guidance is to tailor this information to the patient's relative risk. The definite risk of SUDEP, although small, should be addressed (NICE, 2018).

Failure to address this complex and sensitive issue immediately undermines the ability of patients and their care networks to proactively manage their epilepsy and mitigate SUDEP risk. Early discussion of SUDEP can aid the grieving process of people who have subsequently lost loved ones (Flannery and Lomke, 2018).

Raising awareness of SUDEP, by a neurologist or nurse specialist, should therefore occur early, in accordance with best practise. A SUDEP checklist created by Shankar et al (2018) is one possible way to facilitate this. The checklist outlines modifiable and nonmodifiable risk factors, and possible interventions, with the key focus being on how SUDEP is communicated. Implementation of this checklist significantly reduces seizure frequency and influences sleeping positions in patients with epilepsy, with an overall reduction in participant SUDEP risk.

The checklist provides a structured format that initiates healthy conversation, identifies individual risk, and leads to proactive patient involvement. It also provides clinicians with the appropriate language to engage in a difficult but necessary conversationsomething that research has identified as a sticking point in SUDEP awareness (Keddie et al, 2016). The potential impact of this simple intervention is ample justification for it to be $\{\mathrm{AQ} 20$ : Ed@K $\}$ implemented more widely.

An optimal pharmacological regimen should be established to control seizure frequency and severity (Keddie et al, 2016). However, in practice this is difficult to achieve in all patients, especially given that treatment efficacy decreases with each additional AED (Smith et al, 2015b). AED polypharmacy is a known risk factor for SUDEP. It is therefore the role of the physician and, increasingly in the UK, the nurse specialist to prescribe and review medications (Goodwin et al, 2011). This should take into account patient preference, as this promotes holistic care (Goodwin et al, 2011) and increases patient empowerment (Dins et al, 2018). To accompany this, education on the importance of medication compliance should be provided - a responsibility for all nursing staff.

Given the links that exist between epilepsy, QoL and mental health (Dhaelis, 2018), it is the responsibility of all nurses to assess the mental health of their patients with epilepsy. There is an association between poor mental health and poor medication compliance (Welsh and Kerley, 2009), which in individuals with epilepsy is a substantial risk factor for SUDEP (D) wn et al, 2018). Early identification and treatment or poor mental health in people with epilepsy would help to prevent poor medication compliance, promote better overall health and contribute to improving the QoL of the patient (Lu and Elliott, 2012). This would enable them to 'live better' with epilepsy and, in turn, would help to reduce the risk of a SUDEP event (NICE, 2015).

We should not assume that we know how patients feel about their condition, or that we know best about how to manage it for that individual. Patients should be given permission and space to talk, to inform their decisions about subsequent treatment. This ranges from simple online or community support to formal psychological assessment and management (Smith et al, 2015a).

Existing guidance states that all epilepsy patients should have access to an epilepsy specialist nurse (ESN) C CE, 2018) Access to an ESN allows for the provisturr of holistic, person-centred care. It provides the patient with a direct point of access to voice concerns and continue to proactively manage their 


\section{KEY POINTS}

- Sudden unexpected death in epilepsy (SUDEP) is a major cause of death in individuals with epilepsy

- SUDEP risk factors include poor seizure control, particularly of tonic-clonic seizures, polypharmacy of anti-epileptic drugs and nocturnal seizures

- The underlying pathophysiology of SUDEP is poorly understood, but is due in part to cardiorespiratory dysfunction

nose re are no current treatments for SUDEP, although and regular conversations with patients are beneficial to ensuring that the overall risk can be reduced

condition alongside specialist support. Such access helps to underpin all that is mentioned above, as they $\{A Q 21:$ the $\mathrm{E} D\}$ are best placed to refer patients to additional services beyond their remit.

Education should be viewed as the underpinning component of managing SUDEP risk. Our responsibility as nurses is to educate our patients on ways to optimise their everyday health through health promotion. Modifiable risk factors associated with SUDEP, for instance poor medication compliance, seizure frequency, alcohol consumption, mental health and sleep should be addressed (Brown et al, 2013). Standard epilepsy advice regarding sleep and alcohol consumption should continue in line with best practice guidelines, given that these factors contribute to increased seizure activity and could therefore contribute to an increased risk of unexpected death (Smith et al, 2015a).

Advice and resources should also be provided. Charity organisations such as Epilepsy Action (www. epilepsy.org.uk) and SUDEP Action (www.sudep. org) provide a way for patients, families and carers to educate themselves on the condition and become part of a supportive community. This should not replace the need for face-to-face conversation or education, but should complement ongoing care provision (Keddie et al, 2016).

Primary care and specialist nurses may be best placed to provide this education as they have often built a trusting rapport with the patients they see regularly. Acute care nurses still have the opportunity and responsibility to identify some of these issues if previous engagements have failed to do so, ensuring that fewer patients 'slip through the net'.

This of course relies on nursing staff themselves being effectively educated in epilepsy and SUDEP. There is potential scope here for ESNs to conduct (7) d-based training on the links between these two

\section{CPD reflective questions}

- Having read the potential mechanisms for SUDEP, consider some simple changes in your area of practice that could help to reduce a patient's risk of sudden death? Think about how you might implement them?

(1) ardless of specialty, look back through the 2ronsing responsibilities proposed in this article and think about the following two points. Is enough done in your ward area to discuss epilepsy, SUDEP and the impact on patient quality of life? How could you contribute to the conversation?

- How might you facilitate the development of your knowledge, and also that of others, surrounding epilepsy and SUDEP to improve your practice?

subjects. Enhanced knowledge in nursing staff may increase confidence in addressing some of the issues laid down in this article. This ultimately benefits their patients who themselves become better educated and potentially empowered to take control of their health and reduce their SUDEP risk.

The responsibilities outlined above lead to increased patient and care network knowledge and understanding, improved mental health, increased treatment compliance and subsequently fewer impatient (20) hissions (Buelow et al, 2018). In combination, these strategies could help to reduce SUDEP risk, due to improved epilepsy management, and could be optimised through the SUDEP checklist.

While the role of the non-specialised nurse may seem IIInited they do in fact have a vital role to play as part of the wider multi-disciplinary team $\{A Q 22: \mathrm{Ed} \Omega \mathrm{K}$ ? $\}$ to facilitate care. Research suggests better outcomes when nurses are involved in epilepsy management, regardless of specialisation (Buelow et al, 2018).

\section{Conclusions}

SUDEP is a major cause of death in individuals with epilepsy. There is no current consensus on the underlying pathophysiological causes of SUDEP and further preclinical and clinical research is required. Furthermore, as there is no definitive method to reduce the SUDEP risk, management of the individual at risk is key. All nursing staff, regardless of specialty, through the use of tools such as the SUDEP checklist, can enable this (1) hagement of risk. The skills and attributes required to manage SUDEP risk are not specific; adherence to the NMC $\{\mathrm{AQ} 23:$ Nursing and Midwifery Code? Please supply reference $\}$ code should naturally pave the way to effective, holistic and person-centred care and need only be applied in the context of epilepsy $\{$ AQ24: Please explain the last bit of this sentence\}. Given the overall risk and prevalence of SUDEP, expansion of the knowledge of epilepsy and SUDEP within the nursing profession is a key process through which management 
of risk can be achieved.

\{AQ25: One of your reviewers has said: $\Omega$

I found this article very informative, easy to read and follow, and believe it will be of interest to many readers. The risk of SUDEP is a very contemporary issue, and this article addresses the matter in a way that is applicable to both specialist epilepsy teams as well as more generic health care teams. I was particularly impressed with the excellent use of very up-to-date references and sources of practice-based evidence.

Some suggestions:

- I would suggest that your reflective question no2 asks "is enough done by your team" rather than "in your ward" to make this inclusive of a wider range of health services rather than just hospital ward-based services. Nurses working across the whole range of health care may come across patients who are facing a SUDEP risk.

- You mention the importance of genes that can lead to long QT syndrome. I think a very brief explanation of what long QT syndrome is would be helpful.

- You should write EEG out in full. < [electroencephalogram? Brian]>

- You again make a reference to wards with regards to the receipt of training, and again you are excluding community-based teams and services.

- You write "while the role of the non-specialised nurse may seem limited...'. I think you mean that the role is limited with regards to the delivery of epilepsy care and treatment.

With these few alterations made, my opinion is that this article will be ready for publication.\}

\{AQ26: The other of your reviewers has said:

Very interesting, thought-provoking article which should be of interest to all nurses, especially those working in neurosciences. Potential modes of action that may lead to SUDEP very relevant and explained well, with the addition of potential role of genetics.

The following are suggestions that may help clarify issues for readers:

- Points: 1. Consider rewording as SUDEP is a Tare cause of death and the present wording does not reflect this. Page 11 you mention risk is small. 4. ? may result in a reduction rather than can. $<[\mathrm{By}$ page 11, reviewer means end of first paragraph under the heading Reduction of the likelihood of SUDEP occurring: nursing responsibilities. I think 4 . refers to the last of the Key points. Brian]>

- lective Questions: 2 - clinical area rather than ward as information can be used in all clinical areas including primary / residential.

3rd paragraph - ? uncontrolled epilepsy or seizures (a) er than just epilepsy as majority of people with cprlepsy are seizure free so poor mental health etc is not applicable to all. Last sentance ? reword as present nurses with knowledge ? information / data may be more appropriate. Also review use of word develop twice.

(1) dence rates -page 4 2nd paragraph - Review every person as not all patients are at risk as those with only myoclonic or only absences seizures are not known to be at risk (ref SUDEP.org) - Also consider rewarding Risk Factors - As all individuals are at risk. $<[$ I think this refers to section Incidence rates and prevalence, start of second paragraph. Brian]>

- Risk factors list: 6th - being in bed - consider rewording as sleeping at any time - sofa in afternoon -SUDEP has occurred. You may wish to consider adding in alcohol / substance abuse, depression \& lack of observation ( being alone).

(5) ample of a section of, or the SUDEP Shanker Clal checklist, would enhance the article further $<$ [Perhaps send us a table for inclusion? Brian] $>$.\}

BJNN

Declaration of interest: The authors declare no conflicts of interest.

owledgements: The authors would like to thank Victoria Cox for ritical reading of the manuscript.

Ackland GL, Kasymov V, Gourine AV. Physiological and pathophysiological roles of extracellular ATP in chemosensory control of breathing. Biochem Soc Trans. 2007;35(Pt 5):1264 1268. https://doi.org/10.1042/BST0351264

Bagnall RD, Crompton DE, Semsarian C. Genetic basis of sudden unexpected death in epilepsy. Front Neurol. 2017;8:348. https:// doi.org/10.3389/fneur.2017.00348

Bateman LM, Li C-S, Lin T-C, Seyal M. Serotonin reuptake inhibitors are associated with reduced severity of ictal hypoxemia in medically refractory partial epilepsy. Epilepsia. 2010;51(10):2211-2214. https://doi. org/10.1111/j.1528-1167.2010.02594.x

Brown S, Shankar R, Cox D, McLean MB, Jory C. Clinical governance: risk assessment in SUDEP. Clinical Governance: An International Journal. 2013;18(4):325-331. https://doi. org/10.1108/CGIJ-12-2012-0045

Buchanan GF, Murray NM, Hajek MA, Richerson GB. Serotonin neurones have anti-convulsant effects and reduce seizure-induced mortality. J Physiol. 2014;592(19):4395-4410. https://doi. org/10.1113/jphysiol.2014.277574

Budde RB, Arafat MA, Pederson DJ, Lovick TA, Jefferys JGR, Irazoqui PP. Acid reflux induced laryngospasm as a potential mechanism of sudden death in epilepsy. Epilepsy Res. 2018;148:23-31. https://doi.org/10.1016/j.eplepsyres.2018.10.003

Buelow J, Miller W, Fishman J. Development of an epilepsy nursing communication tool: Improving the quality of interactions between nurses and patients with seizures. J Neurosci Nurs. 2018;50(2):74-80. https://doi.org/10.1097/ JNN.0000000000000353

Cipriani A, Furukawa TA, Salanti G et al. Comparative efficacy and acceptability of 21 antidepressant drugs for the acute treatment of adults with major depressive disorder: a systematic review and network meta-analysis. Lancet.

2018;391(10128):1357-1366. https://doi.org/10.1016/S0140- 
6736(17)32802-7

Cubeddu LX. Drug-induced inhibition and trafficking disruption of ion channels: pathogenesis of QT abnormalities and druginduced fatal arrhythmias. Curr Cardiol Rev. 2016;12(2):141154. PMID: 26926294

Ead H. Review of laryngospasm and noncardiogenic pulmonary edema. Dynamics. 2003;14(3):9-12. PMID: 14725141

Ellis SP, Szabo CA. Sudden unexpected death in epilepsy: incidence, risk factors, and proposed mechanisms. Am J Forensic Med Pathol. 2018;39(2):98-102. https://doi.org/10.1097/ PAF.0000000000000394

Fisher RS, Acevedo C, Arzimanoglou A et al. ILAE official report: a practical clinical definition of epilepsy. Epilepsia. 2014;55(4):475-482. https://doi.org/10.1111/epi.12550

Fisher RS, Nune G, Roberts SE, Cramer JA. The Personal Impact of Epilepsy Scale (PIES). Epilepsy Behav. 2015;42:140-146. https://doi.org/10.1016/j.yebeh.2014.09.060

Flannery RB, Lomke E. Sudep and grief: overview and current issues. Psychiatr Q. 2018. https://doi.org/10.1007/s11126-0189608-1

Friedman D, Kannan K, Faustin A et al. Cardiac arrhythmia and neuroexcitability gene variants in resected brain tissue from patients with sudden unexpected death in epilepsy (SUDEP). NPJ Genom Med. 2018;3:9. https://doi.org/10.1038/s41525-0180048-5

Goodwin M, Higgins S, Lewis S. Epilepsy specialist nurse prescribing practice in the United Kingdom: A national questionnaire survey. Seizure. 2011;20(10):754-757. https://doi org/10.1016/j.seizure.2011.07.004

Hammers A, Asselin M-C, Hinz R et al. Upregulation of opioid receptor binding following spontaneous epileptic seizures. Brain. 2007;130(4):1009-1016. https://doi.org/10.1093/brain/awm012

Higgins A, Downes C, Varley J et al. Patients with epilepsy care experiences: comparison between services with and without an epilepsy specialist nurse. Epilepsy Behav. 2018;85:85-94. https:// doi.org/10.1016/j.yebeh.2018.05.038

Hodges MR, Wehner M, Aungst J, Smith JC, Richerson GB. Transgenic mice lacking serotonin neurons have severe apnea and high mortality during development. J Neurosci. 2009;29(33):10341-10349. https://doi.org/10.1523/ JNEUROSCI.1963-09.2009

Kavitt RT, Vaezi MF. Laryngpharyngeal reflux. In Vela MF, Richter JE, Pandolfino JE (eds). Practical manual of gastroesophageal reflux disease. John Wiley \& Sons; 2013;pp154 AQ27: Please ensure that I have edited this reference d 2 ty

Keddie S, Angus-Leppan H, Parker T et al. Discussing sudden unexpected death in epilepsy: are we empowering our patients? A questionnaire survey. JRSM Open. 2016;7(9):2054270416654358. https://doi. org/10.1177/2054270416654358

Kennedy JD, Hardin KA, Parikh P, Li C-S, Seyal M. Pulmonary edema following generalized tonic clonic seizures is directly associated with seizure duration. Seizure. 2015;27:19-24. https:// doi.org/10.1016/j.seizure.2015.02.023

Lacuey N, Vilella L, Hampson JP, Sahadevan J, Lhatoo SD. Ictal laryngospasm monitored by video-EEG and polygraphy: a potential SUDEP mechanism. Epileptic Disord. 2008;20(2):146150. https://doi.org/10.1684/epd.2018.0964

Loughlin CJ, Koufman JA, Averill DB et al. Acid-induced laryngospasm in a canine model. Laryngoscope. 1996;106(12 Pt 1):1506-1509. PMID: 8948612

Lu B, Elliott JO. Beyond seizures and medications: Normal activity limitations, social support, and mental health in epilepsy. Epilepsia. 2012;53(2):e25-e28. https://doi. org/10.1111/j.1528-1167.2011.03331.x

Maguire MJ, Jackson CF, Marson AG, Nevitt SJ. Treatments for the prevention of sudden unexpected death in epilepsy (SUDEP) Cochrane Database Syst Rev. 2016;7:CD011792. https://doi. org/10.1002/14651858.CD011792.pub2

Michaelis R, Tang V, Goldstein LH. Psychological treatments for adults and children with epilepsy: evidence-based recommendations by the International League Against Epilepsy Psychology Task Force. Epilepsia. 2018;59(7):1282-1302. https:// doi.org/10.1111/epi.14444

Middleton O, Atherton D, Bundock E et al. National Association of Medical Examiners position paper: recommendations for the investigation and certification of deaths in people with epilepsy. Epilepsia. 2018;59(3):530-543. https://doi.org/10.1111/epi.14030

Mula M, Sander JW. Psychosocial aspects of epilepsy: a wider approach. BJPsych Open. 2016;2(4):270-274. https://doi. org/10.1192/bjpo.bp.115.002345

Myers K, Bello-Espinosa L, Symonds J et al. Heart rate variability in epilepsy: a potential SUDEP risk biomarker (p6.263). Neurology. 2018;90(15 Supplement). http://n.neurology.org/ content/90/15_Supplement/P6.263 (accessed 20 February 2019)

Nakase K, Kollmar R, Lazar J et al. Laryngospasm, central and obstructive apnea during seizures: defining pathophysiology for sudden death in a rat model. Epilepsy Res. 2016;128:126-139. https://doi.org/10.1016/j.eplepsyres.2016.08.004

Nashef L, So EL, Ryvlin P, Tomson T. Unifying the definitions of sudden unexpected death in epilepsy. Epilepsia. 2012;53(2):227233. https://doi.org/10.1111/j.1528-1167.2011.03358.x

National Institute for Health and Care Excellence. Epilepsies: Diagnosis and management [CG137]. 2018. https://www.nice. org.uk/guidance/cg137 (accessed 20 February 2019)

National Institute for Health and Care Excellence. The epilepsies: the diagnosis and management of the epilepsies in adults and children in primary and secondary care [CG20]. 2015. https:// www.nice.org.uk/guidance/cg20 (accessed 20 February 2019)

Pattinson KT. Opioids and the control of respiration. Br J Anaesth. 2008;100(6):747-758. https://doi.org/10.1093/bja/aen094

Public Health England. Deaths associated with neurological conditions in england 2001 to 2014 data analysis report. 2018. https://tinyurl.com/y7dxg2ol (accessed 20 February 2019)

Rheims S, Valton L, Michel V et al. Efficacy of naloxone in reducing postictal central respiratory dysfunction in patients with epilepsy: study protocol for a double-blind, randomized, placebo-controlled trial. Trials. 2016;17(1):529. https://doi. org/10.1186/s13063-016-1653-1

Ryvlin P, Nashef L, Lhatoo SD et al. Incidence and mechanisms of cardiorespiratory arrests in epilepsy monitoring units (MORTEMUS): a retrospective study. Lancet Neurol. 2013;12(10):966-977. https://doi.org/10.1016/S14744422(13)70214-X

Shankar R, Henley W, Boland C, et al. Decreasing the risk of sudden unexpected death in epilepsy: structured communication of risk factors for premature mortality in people with epilepsy. Eur J Neurol. 2018;25(9):1121-1127. https://doi.org/10.1111/ ene. 13651

Smith G, Wagner JL, Edwards JC. CE: Epilepsy update, part 1: refining our understanding of a complex disease. Am J Nurs. 2015a;115(5):40-47, quiz 48-49. https://doi.org/10.1097/01. NAJ.0000465030.89975.e8

Smith G, Wagner JL, Edwards JC. Epilepsy update, part 2 nursing care and evidence-based treatment. Am J Nurs. 2015b;115(6):34 44. https://doi.org/10.1097/01.NAJ.0000466314.46508.00

Stewart M, Kollmar R, Nakase K et al. Obstructive apnea due to laryngospasm links ictal to postictal events in SUDEP cases and offers practical biomarkers for review of past cases and prevention of new ones. Epilepsia. 2017;58(6):e87-e90. https:// doi.org/10.1111/epi.13765

Tavee J, Morris III H. Severe postictal laryngospasm as a potential mechanism for sudden unexpected death in epilepsy: a near-miss in an EMU. Epilepsia. 2008;49(12):2113-2117. https://doi. org/10.1111/j.1528-1167.2008.01781.x

Teran FA, Massey CA, Richerson GB. Serotonin neurons and central respiratory chemoreception: where are we now? Prog Brain Res. 2014;209:207-233. https://doi.org/10.1016/B978-0444-63274-6.00011-4

Tester DJ, Ackerman MJ. Genetics of long QT syndrome. Methodist DeBakey Cardiovasc J. 2014;10(1):29-33. PMID: 24932360

Thurman DJ, Hesdorffer DC, French JA. Sudden unexpected death in epilepsy: assessing the public health burden. Epilepsia. 2014;55(10):1479-1485. https://doi.org/10.1111/epi.12666 Tiron C, Campuzano O, Pérez-Serra A et al. Further evidence of 
the association between LQT syndrome and epilepsy in a family with KCNQ1 pathogenic variant. Seizure. 2015;25:65-67. https:// doi.org/10.1016/j.seizure.2015.01.003

Tupal S, Faingold CL. Evidence supporting a role of serotonin in modulation of sudden death induced by seizures in DBA/2 mice. Epilepsia. 2006;47(1):21-26. https://doi. org/10.1111/j.1528-1167.2006.00365.x

Velagapudi P, Turagam M, Laurence T, Kocheril A. Cardiac arrhythmias and sudden unexpected death in epilepsy (SUDEP). Pacing Clin Electrophysiol. 2012;35(3):363-370. https://doi. org/10.1111/j.1540-8159.2011.03276.x

Watkins L, Shankar R, Sander JW. Identifying and mitigating sudden unexpected death in epilepsy (SUDEP) risk factors. Expert Rev Neurother. 2018;18(4):265-274. https://doi.org/10.10 80/14737175.2018.1439738

Welsh R, Kerley S. Nursing patients with epilepsy in secondary care settings. Nurs Stand. 2009;23(36):49-56; quiz 58, 60. https://doi. org/10.7748/ns2009.05.23.36.49.c6970 